\title{
IMAGING SYSTEMS FOR SIZE MEASUREMENTS OF DEBRISAT FRAGMENTS
}

\author{
B. Shiotani, T. Scruggs, R. Toledo ${ }^{1}$

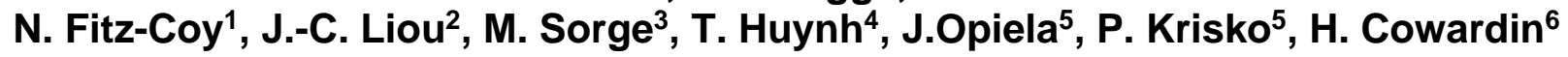 \\ ${ }^{1}$ UF, ${ }^{2}$ NASA, ${ }^{3}$ The Aerospace Corporation, ${ }^{4}$ AF/SMC, ${ }^{5}$ Jacobs, ${ }^{6}$ UTEP-Jacobs JETS
}

The overall objective of the DebriSat project is to provide data to update existing standard spacecraft breakup models. One of the key sets of parameters used in these models is the physical dimensions of the fragments (i.e., length, average-cross sectional area, and volume). For the DebriSat project, only fragments with at least one dimension greater than $2 \mathrm{~mm}$ are collected and processed. Additionally, a significant portion of the fragments recovered from the impact test are needle-like and/or flat plate-like fragments where their heights are almost negligible in comparison to their other dimensions. As a result, two fragment size categories were defined: 2D objects and 3D objects. While measurement systems are commercially available, factors such as measurement rates, system adaptability, size characterization limitations and equipment costs presented significant challenges to the project and a decision was made to develop our own size characterization systems. The size characterization systems consist of two automated image systems, one referred to as the 3D imaging system and the other as the 2D imaging system. Which imaging system to use depends on the classification of the fragment being measured. Both imaging systems utilize point-and-shoot cameras for object image acquisition and create representative point clouds of the fragments. The $3 \mathrm{D}$ imaging system utilizes a space-carving algorithm to generate a 3D point cloud, while the 2D imaging system utilizes an edge detection algorithm to generate a 2D point cloud. From the point clouds, the three largest orthogonal dimensions are determined using a convex hull algorithm. For 3D objects, in addition to the three largest orthogonal dimensions, the volume is computed via an alpha-shape algorithm applied to the point clouds. The average cross-sectional area is also computed for 3D objects. Both imaging systems have automated size measurements (image acquisition and image processing) driven by the need to quickly and accurately measure tens of thousands of debris fragments. Moreover, the automated size measurement reduces potential fragment damage/mishandling and ability for accuracy and repeatability. As the fragment characterization progressed, it became evident that the imaging systems had to be revised. For example, an additional view was added to the 2D imaging system to capture the height of the 2D object. This paper presents the DebriSat project's imaging systems and calculation techniques in detail; from design and development to maturation. The experiences and challenges are also shared. 